volunteers. In the event of deterioration with escalation of severity category repeat samples were obtained.

Results Patients with COVID-19 were more likely to be male (67\% vs $20 \%(\mathrm{HC}) ; \mathrm{p}<0.001)$, older $(64.4 \pm 16.7$ vs $47.7 \pm$ $13.5 ; \mathrm{p}<0.001)$ have a greater BMI $(32.3 \pm 6.6$ vs $27.9 \pm$ $5.1 ; \mathrm{p}=0.01)$ and be never-smokers $(60 \%$ vs $30 \% ; \mathrm{p}=0.001)$. We demonstrated a hyperinflammatory and pro-coagulative state in all patients with COVID-19. All measures of complement activity were significantly higher in patients with COVID-19, including levels of C5a (HC 13[7,21] vs Covid-19 $35[24,43] ; \mathrm{p}<0.001$ ) and SC5b9-complex (HC 654[419, 1120] vs Covid-19 1452[970, 2170]; p<0.001) which both increased with disease severity and were statistically significantly different between mild and severe disease. SC5b9-complex was significantly higher in patients who deteriorated from moderate to severe disease (1393 [1019, 1986] vs 2116 [958, 4538]; $\mathrm{p}=0.03)$.

Discussion Our findings demonstrated increased levels of complement activity in patients with COVID-19, particularly in those patients requiring non-invasive and mechanical ventilation and those patients that deteriorate requiring increasing ventilatory support. The complement cascade is a key player in protective immunity against pathogens, with its activation orchestrating key immunoprotective and anti-inflammatory effects. Increased activation of the complement cascade may contribute to the dysregulated and destructive inflammatory response that leads to multi-organ failure and our findings suggest a potentially important treatment target for COVID-19.

\section{S57 EXPRESSION OF THE SARS-COV-2 RECEPTORS ACE2 AND TMPRSS2 IN THE RESPIRATORY TRACT OF CHILDREN AND ADULTS}

AG Nuttall, C Hedrich, C Semple, P Losty, R Shukla, J McPartland, S Williams, S Northey, P McNamara. University of Liverpool, Liverpool, UK

\subsection{6/thorax-2021-BTSabstracts.63}

Background Angiotensin-converting enzyme 2 (ACE2) receptors and the serine protease co-factor TMPRSS2 are cellular receptors/co-factors for SARS-CoV2, allowing viral entry into host cells. Children, when infected with SARS-CoV2, generally present with mild disease, and particularly milder lower respiratory tract symptoms. One hypothesis to explain this phenomenon is differential expression of ACE2 and TMPRSS2 in the respiratory tracts of children and adults.

Aims To investigate ACE2 receptor and TMPRSS2 expression in upper/lower respiratory tracts of children and adults without COVID disease.

Methods Nasal brushings from obtained from children of different ages (3 months -15 years) undergoing routine elective surgery, and from volunteer adults (20-61 years). Nasal epithelial ACE2 and TMPRSS2 mRNA expression was analysed by PCR.

Post-mortem lung tissue from children and adults without COVID-19 was stained to identify ACE2 and TMPRSS2 protein expression by immunohistochemistry (IHC). Each sample was digitalised using Philips Digital Pathology Solutions software, with three alveolar and three bronchial screen-grab images obtained at $\mathrm{x} 40$ magnification, and analysed using Image $\mathrm{J}$.

Results Nasal ACE2 and TMPRSS2 mRNA expression in children and adolescents $(n=12)$ and adults $(n=26)$ was similar. Immunohistochemical lung tissue ACE2 and TMPRSS2 protein

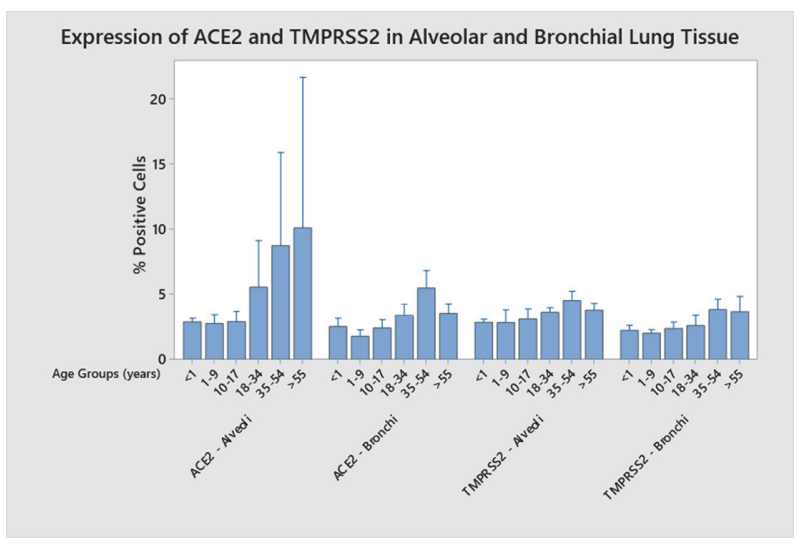

Abstract S57 Figure 1 Bar chart with error bars displaying mean $\%$ positive cells for each age group for alveolar and bronchial ACE2 and TMPRSS2

expression in 38 subjects $(<1 \mathrm{yr} n=9,1-9 \mathrm{yrs} n=4,10-17 \mathrm{yrs}$ $\mathrm{n}=6,18-34 \mathrm{yrs} \mathrm{n}=7,35-54 \mathrm{yrs} \mathrm{n}=7,>55 \mathrm{yrs} \mathrm{n}=5$ ) was significantly greater in alveolar than bronchial sections. In children, ACE2 and TMPRSS2 expression was detected in only 2-3\% of cells in alveolar and bronchial tissue. Alveolar ACE2 receptor expression was significantly greater in adults and appeared to increase with age. Adult alveolar ACE2 receptor expression was highly variable, being detected in some specimens in $\sim 30 \%$ of cells.

Conclusion ACE2 and TMPRSS2 expression is similar in upper airways of children and adults, likely indicating that both groups are equally susceptible to SARS-CoV2 infection. In contrast, expression of both these receptors/co-factors is greater in adult lower airways of adults, and particularly for ACE2 receptor in alveolar tissue. In some adults, ACE2 receptor was detected in up to a quarter of alveolar cells, potentially explaining why some adults are so susceptible to lower respiratory tract disease.

\section{Treatment choices in cystic fibrosis and bronchiectasis: what works and when}

\section{S58 TRIPLE CFTR MODULATORS IMPROVE SINO-NASAL AND LARYNGOPHARYNGEAL REFLUX SYMPTOMS IN PEOPLE WITH ADVANCED CYSTIC FIBROSIS LUNG DISEASE}

${ }^{1} \mathrm{~S}$ Shakir, ${ }^{1} \mathrm{C}$ Echevarria, ${ }^{1} \mathrm{~S}$ Doe, ${ }^{2} \mathrm{M}$ Brodlie, ${ }^{2} \mathrm{C}$ Ward, ${ }^{2} \mathrm{~S} J$ Bourke. ${ }^{1}$ Adult Cystic Fibrosis Centre, Royal Victoria Infirmary, Newcastle upon Tyne, UK; ${ }^{2}$ Translational and Clinical Research Institute, Newcastle University, Newcastle upon Tyne, UK

\subsection{6/thorax-2021-BTSabstracts.64}

Background Triple CFTR modulator therapy (elexacaftor/tezacaftor/ivacaftor) improves lung function, weight, exacerbation rates and quality of life in people with Cystic Fibrosis. CF is a multisystem disease and there is increasing interest in the extrapulmonary effects of CFTR modulators. Chronic rhinosinusitis and gastroesophageal reflux are common in people with CF and cause a high level of sino-nasal and laryngopharyngeal symptoms. We assessed the effect of triple CFTR modulator therapy on these symptoms in a cohort of patients with advanced CF lung disease.

Method In a prospective study, we used the Sino-Nasal Outcome Test (SNOT), the Reflux Symptom Index (RSI) and the 
Abstract S58 Table 1 Measured values at baseline and after 6 months' treatment.

\begin{tabular}{llll}
\hline & Baseline & 6 months & Difference \\
\hline ppFEV1 $^{1}$ & $24.8(7.13)$ & $33.4(10.33)$ & $8.6^{3}$ \\
BMI $^{1}$ & $21.3(4.13)$ & $23.9(4.29)$ & $2.6^{3}$ \\
RSI $^{2}$ & $15(10.75-23)$ & $5(2.25-7)$ & $10^{3}$ \\
HARQ $^{2}$ & $26.5(16-39)$ & $7(3.75-12.25)$ & $19.5^{3}$ \\
SNOT-20 $^{2}$ & $36.5(22-42)$ & $20(10-31.25)$ & $16.5^{3}$ \\
\hline
\end{tabular}

${ }^{1}$ mean (Standard Deviation)

2 median (IQR)

${ }^{3} \mathrm{p}<0.001$

ppFEV1 - percentage predicted Forced Expiratory Volume in 1 second, BMI - Body Mass Index, RSI - Reflux Symptom Index, HARQ - Hull Airway Reflux Questionnaire, SNOT-20 Sino-Nasal Outcome Test 20

Hull Airway Reflux Questionnaire (HARQ) as patient-reported outcome measures (PROMs) to assess the effect of triple CFTR modulators on sino-nasal and laryngoesophageal reflux symptoms. Questionnaires, lung function and weight were recorded at baseline before starting treatment and after 6 months on treatment.

Results 32 patients (23 male) starting elexacaftor/tezacaftor/ivacaftor were studied. Their baseline characteristics were mean age 34.3 (range 20-65) years, $\mathrm{FEV}_{1} \%$ predicted 24.8 (11-40), weight $63.2 \mathrm{~kg}(35-99.8)$ and BMI $21.28 \mathrm{~kg} / \mathrm{m}^{2}$ (13.2-31.1). All patients continued with treatment throughout the study period. At 6 months there was an improvement in mean FEV1\% predicted of 8.63 and BMI $2.6 \mathrm{~kg} / \mathrm{m}^{2}$. Patient reported outcome measures showed significant improvement (table 1): median scores RSI 10, HARQ 19.5 and SNOT 16 $(\mathrm{p}<0.001$ for all outcomes).

Discussion This study shows significant improvement in lung function, weight and sino-nasal and laryngopharyngeal reflux PROMs in patients with advanced CF. The SNOT-20, RSI and HARQ scores showed improvement that exceeded recognised clinically significant changes in these metrics.

\section{S59 ADHERENCE TO NEBULISED THERAPIES IN PEOPLE WITH CYSTIC FIBROSIS STARTING ELEXACAFTOR/ TEZACAFTOR/IVACAFTOR (KAFTRIO)}

IB Howell, A Tugwell, D Bhaskaran, NJ Bell. Bristol Adult Cystic Fibrosis Centre, Bristol, UK

\subsection{6/thorax-2021-BTSabstracts.65}

Introduction Cystic Fibrosis HealthHub (CFHH) is a digital platform in use by 17 adult CF centres in the UK which improves patient self-care by objectively monitoring adherence to nebulised therapies delivered via e-Track nebulisers which record device usage on a central server. This study aimed to objectively measure a perceived decrease in adherence with nebulised therapies following initiation of the Elexacaftor-Tezacaftor-Ivacaftor oral CFTR modulator therapy.

Methods We identified all patients on Kaftrio currently enrolled in $\mathrm{CFHH}$ who regularly uploaded data. We compared average CFHH-measured adherence 3 months before and after starting Kaftrio. We reviewed documentation on our clinical database of any patient or healthcare professional decision to change nebuliser usage.

Results 154 patients were taking Kaftrio. 71 were not enrolled in $\mathrm{CFHH}, 34$ did not regularly upload data, and 2 had been instructed to change therapy during the study period leaving

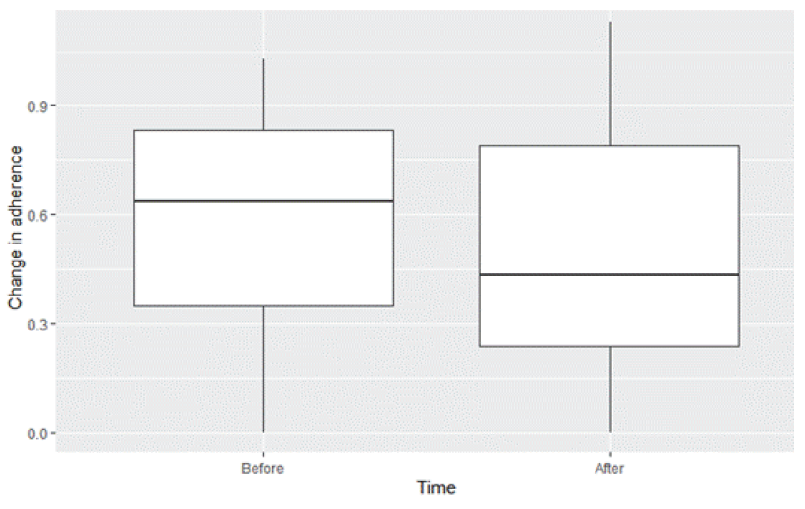

Abstract S59 Figure 1 Box plot comparing average adherence of nebulised therapy before and after Kaftrio initition

47 patients included in analysis. 31 patients (65\%) reduced their adherence to nebulised therapies following Kaftrio use. Median nebulised therapy adherence dropped from $65 \%$ to $42 \%(\mathrm{p}<0.003$, Wilcoxson Signed Rank) pre and post Kaftrio introduction respectively (figure 1 ). Of the 47 patients, 28 (60\%) communicated a decision to change therapy with the CF team, while 19 (40\%) did not communicate this change.

Discussion Our data demonstrates a reduction in nebulised therapy adherence after Kaftrio initiation. Decisions to reduce adherence were often patient driven and not disclosed to clinicians.

Our findings underline the importance of including objective measures of adherence to inhaled therapies in the design of CFTR modulator studies.

The lack of CFHH uploads for 34 patients highlights the challenges in monitoring adherence in clinical practice; in our experience, these patients were less adherent to treatment.

We plan to conduct a qualitative study to explore factors influencing patient decisions to stop or continue medication.

\section{S60 OBSERVATIONAL STUDY OF IVACAFTOR IN PEOPLE WITH CYSTIC FIBROSIS AND SELECTED NON-G551D GATING MUTATIONS: FINAL RESULTS FROM VOCAL}

${ }^{1} \mathrm{NJ}$ Simmonds, ${ }^{2} \mathrm{~K}$ van der Ent, ${ }^{3} \mathrm{C}$ Colombo, ${ }^{4} \mathrm{~N}$ Kinnman, ${ }^{4} \mathrm{C}$ DeSouza, ${ }^{4} \mathrm{~T}$ Thorat, ${ }^{4} \mathrm{~K}$ Chandarana, ${ }^{5} \mathrm{C}$ Castellani. ${ }^{1}$ Adult Cystic Fibrosis Centre, Royal Brompton Hospital and Imperial College London, London, UK; ${ }^{2}$ Department of Pediatric Respiratory Diseases, University Medical Center Utrecht, Utrecht, The Netherlands; ${ }^{3}$ Fondazione IRCCS Ca' Granda Ospedale Maggiore Policlinico, University of Milan, Milan, Italy; ${ }^{4}$ Vertex Pharmaceuticals Incorporated, Boston, USA; ${ }^{5}$ Cystic Fibrosis Centre, IRCCS Istituto Giannina Gaslini, Genoa, Italy

\subsection{6/thorax-2021-BTSabstracts.66}

Introduction and Objectives VOCAL, a Phase 4 observational study (NCT02445053), assessed real-world effectiveness of ivacaftor (IVA) in people with cystic fibrosis (pwCF) with $\geq 1$ non-G551D gating mutation (G178R, S549N, S549R, G551S, G1244E, S1251N, S1255P or G1349D).

Methods PwCF aged $\geq 6$ years in Italy, the Netherlands and the UK who were IVA-naïve or on IVA for $\leq 18$ months at enrolment were eligible. Data were recorded for 12 months pre-IVA and up to 48 months after enrolment. Continuous outcomes (e.g. percent predicted forced expiratory volume in 1 second $\left[\mathrm{ppFEV}_{1}\right]$, body mass index $[\mathrm{BMI}]$ ) were assessed from baseline (the last pre-IVA value recorded) in 6-month intervals up to 48 months post-IVA using a mixed model for 